\title{
Fructose as a carbon source induces an aggressive phenotype in MDA-MB-468 breast tumor cells
}

\author{
BEHJATOLAH MONZAVI-KARBASSI ${ }^{1,6}$, R. JEAN HINE ${ }^{2,6}$, JOSEPH S. STANLEY ${ }^{1}$, VISHNU PRAKASH RAMANI ${ }^{3}$, \\ JAIME CARCEL-TRULLOLS ${ }^{1}$, TRACY L. WHITEHEAD ${ }^{1}$, THOMAS KELLY ${ }^{1,6}$, ERIC R. SIEGEL ${ }^{4}$, \\ CECILE ARTAUD $^{1}$, SAEID SHAAF ${ }^{1}$, RINKU SAHA $^{1}$, FARIBA JOUSHEGHANY ${ }^{1}$, \\ RONDA HENRY-TILLMAN ${ }^{5}$ and THOMAS KIEBER-EMMONS ${ }^{1,6}$
}

\begin{abstract}
Departments of ${ }^{1}$ Pathology, ${ }^{2}$ Geriatrics, ${ }^{3}$ Biochemistry and Molecular Biology, ${ }^{4}$ Biostatistics, ${ }^{5}$ Surgery, and ${ }^{6}$ The Winthrop P. Rockefeller Cancer Institute, University of Arkansas for Medical Sciences, Little Rock, AR 72205, USA
\end{abstract}

Received April 20,2010; Accepted May 28, 2010

DOI: 10.3892/ijo_00000710

\begin{abstract}
Aberrant glycosylation is a universal feature of cancer cells, and certain glycan structures are well-known markers for tumor progression. Availability and composition of sugars in the microenvironment may affect cell glycosylation. Recent studies of human breast tumor cell lines indicate their ability to take up and utilize fructose. Here we tested the hypothesis that adding fructose to culture as a carbon source induces phenotypic changes in cultured human breast tumor cells that are associated with metastatic disease. MDA-MB-468 cells were adapted to culture media in which fructose was substituted for glucose. Changes in cell surface glycan structures, expression of genes related to glycan assembly, cytoskeleton F-actin, migration, adhesion and invasion were determined. Cells cultured in fructose expressed distinct cell-surface glycans. The addition of fructose affected sialylation and fucosylation patterns. Fructose feeding also increased binding of leukoagglutinating Phaseolus vulgaris isolectin, suggesting a possible rise in expression of branching B-1, 6 GlcNAc structures. Rhodamine-phalloidin staining revealed an altered F-actin cytoskeletal system. Fructose accelerated cellular migration and increased invasion. These data suggest that changing the carbon source of the less aggressive MDAMB-468 cell line induced characteristics associated with more
\end{abstract}

Correspondence to: Dr Behjatolah M. Karbassi, Department of Pathology, Slot \#824, Little Rock, AR 72205, USA

E-mail:karbassi@uams.edu

Abbreviations: GLUT-5, hexose transporter protein SLC2A5; GlcNAc, N-acetylglucosamine; GalNAc, N-acetylgalactosamine; PNA, Arachis hypogaea agglutinin; Lotus, Lotus tetragonolobus lectin; SNA, Sambucus nigra agglutinin; MAA, Maackia amurensis agglutinin; ECL, Erythrina cristagalli lectin; ULEX, Ulex europaeus lectin; PHA-L, Phaseolus vulgaris leukoagglutinating

Key words: glycans, breast cancer, fructose, metastasis, MDAMB-468 cells aggressive phenotypes. These data could be of fundamental importance due to the markedly increased consumption of sweeteners containing free fructose in recent years, as they suggest that the presence of fructose in nutritional microenvironment of tumor cells may negatively affect the outcome for some breast cancer patients.

\section{Introduction}

Tumor initiation and progression are associated with modifications in the structures of glycans covalently attached to glycoproteins and glycolipids present at the cell surface (1). Changes in glycosylation patterns affect the functional characteristics of glycoproteins and proteoglycans, leading to changes in the behavior of tumor cells. It is well recognized that aberrant carbohydrate expression is relevant to tumor metastasis and poor prognosis in cancer patients (2-5). Since cell surface carbohydrates are critical in many interactions between tumor cells and their microenvironment, it is timely to focus on factors that may affect glycan composition or structure but have not been explored rigorously.

It has been proposed that dietary sugars have regulatory roles in enzymatic glycosylation reactions in humans (6). In vitro studies in normal cells have consistently shown that exogenous sugars affect cell differentiation and cell-surface glycan structures (7-9). Fructose has emerged as such an important component of human diets that increasing attention is directed to its consequences in health and disease. Overexpression of the high affinity fructose transport protein is observed in human breast tumor cells (10-12). In animal models there is evidence that fructose-fed rodents had a higher incidence of tumors and an increased frequency of metastases than controls (13-15). This indicates the possibility that increased uptake of fructose can modify the surface glycomic profile of tumor cells, leading to the emergence of more metastatic phenotypes.

MDA-MB-468 human breast tumor cells are able to import fructose $(11,16)$ and fructose-feeding has been shown to increase tumor metastases in an animal model (13-15). These findings led us to develop the hypothesis that introducing fructose as a carbon source in the microenvironment of 
mammary tumor cells modifies cell surface carbohydrate structures and affects tumor cell phenotype. Our data suggest that using fructose as a carbon source in tissue culture is associated with phenotypic changes in this prototypic basallike human breast tumor cell MDA-MB-468 that are consistent with selection for an aggressive phenotype. As MDA-MB-468 is a basal-like tumor cell line derived from an African American women $(17,18)$ and fructose sources are prominent in the diet of African Americans (19), it can be speculated that high intake of fructose might exacerbate an aggressive tumor cell phenotype which is a therapeutic challenge for this special population (20).

\section{Materials and methods}

Reagents and supplies. DMEM culture medium, Dulbecco's PBS and other cell culture supplies were purchased from Cellgro Mediatech, Inc. (Herndon, VA). Fetal bovine serum was obtained from Atlas Biologicals (Fort Collins, CO). Invitrolon polyvinylidene difluoride (PVDF) membranes and Bis-Tris NuPAGE gels were obtained from Invitrogen (Carlsbad, CA). Vimentin and E-cadherin antibodies were purchased from R\&D Systems (Minneapolis, MN). HRPconjugated polyclonal anti goat immunoglobulins were ordered from Dako (Carpinteria, CA). ECL Plus reagent was obtained from GE Healthcare (Piscataway, NJ). Pierce Biotechnology (Rockford, IL) was the source of the M-Per lysis buffer. Roche complete protease inhibitor cocktail was obtained from Roche Applied Sciences (Indianapolis, IN). Human umbilical vein endothelial cells (HUVECs) and endothelial cell basal medium were purchased from Cambrex Bio Science Walkersville, Inc. (Walkersville, MD). TNF- $\alpha$, hexoses and BCA assay kits were obtained from Sigma-Aldrich (St. Louis, MO). Calcein AM was purchased from Molecular Probes, Inc. (Eugene, OR). Transwells and cell culture plates were bought from Fisher Scientific (Houston, TX). Matrigel was ordered from Collaborative Biomedical Products (Bedford, MA). DIF-Quick stain was obtained from IMEB, Inc. (San Marcos, CA). Rhodamine phalloidin was purchased from Molecular Probes.

Cells and culture conditions. The MDA-MB-468 human breast adenocarcinoma cell line was a kindly provided by Dr Janet Price, Department of Cancer Biology, University of Texas M.D. Anderson Cancer Center, Houston, TX. The cell line was isolated from an African American woman with adenocarcinoma of the breast. Sugar-free DMEM was used to prepare the cell culture media. The final hexose concentration of all media was $25 \mathrm{mM}$, corresponding to the concentration of glucose in standard cell culture medium formulations. The three test media contained all glucose (glucose), glucose and fructose 1:1 (glucose/fructose), or fructose and glucose 4:1 (fructose) as carbon sources. Media were supplemented with $10 \%$ heat-inactivated fetal bovine serum, L-glutamine, and penicillin/streptomycin. Cells were cultured in a humidified atmosphere of $5 \% \mathrm{CO}_{2} / 95 \%$ air at $37^{\circ} \mathrm{C}$. The Promega CellTiter 96 AQueous One Solution Cell Proliferation Assay was used to assess cell growth. A solution combines PES, a chemically stable electron coupling reagent, and MTS, a novel tetrazolium compound that is soluble in tissue culture medium. Absorbance of the formazan product at $490 \mathrm{~nm}$ was measured. It is directly proportional to the number of living cells in each well. Cellular morphology was observed by phase-contrast microscopy. Cells were adapted to media containing fructose for 21 days before studies were initiated.

Flow cytometry. Cells were seeded into new flasks $24 \mathrm{~h}$ before analysis. The subconfluent monolayer of cells was detached and washed with Dulbecco's PBS. Cells were transferred to fluorescence-activated cell-sorting (FACS) buffer (Dulbecco's PBS, $1 \%$ bovine serum albumin, and $0.1 \%$ sodium azide), counted, and diluted to $\sim 1-2 \times 10^{6} / \mathrm{ml}$. Biotinylated lectins were added to the cells at a final concentration of $5 \mu \mathrm{g} / \mathrm{ml}$. Cells were incubated on ice for $30 \mathrm{~min}$ and washed twice with FACS buffer before fluorescein isothiocyanate-conjugated streptavidin $(2 \mu \mathrm{g} / \mathrm{ml})$ was added for lectin analysis. Cells were washed and fixed with paraformaldehyde. Acquisition of data was performed by using FACSAria flow cytometer and analysis performed by CellQuest software (both from BD Biosciences, Bedford, MA).

Human umbilical vein endothelial cell (HUVEC) adhesion assay. Clonetics ${ }^{\mathrm{TM}}$ human umbilical vein endothelial cell (HUVEC) system was used (Cambrex Bio Science). Monolayers of HUVEC cells were prepared according to the provider's instructions. Cells were plated in 96-well culture plates at $10^{4}$ cells/well and incubated at $37^{\circ} \mathrm{C}$ in $5 \% \mathrm{CO}_{2}$ for $24 \mathrm{~h}$. After incubation, the HUVECs were treated for $4 \mathrm{~h}$ with $200 \mu \mathrm{g} / \mathrm{ml}$ of tumor necrosis factor- $\alpha$. The tumor cells were pre-labeled with the fluorescent probe (Calcein AM) for $30 \mathrm{~min}$ at $37^{\circ} \mathrm{C}$. These fluorescent cells were incubated with the HUVECs at $37^{\circ} \mathrm{C}$ for $45 \mathrm{~min}$. At the end of the coincubation, non-adherent cells were removed by gentle washing. Fluorescence intensity was measured using the FLx800 MultiDetection Microplate Reader (Bio-Tek Instruments, Inc., Winooski, VT).

Cell and migration assays. Cellular migration was assessed by the wound-healing and transwell assays. For woundhealing assay, cells in complete medium were seeded into a 24-well culture plate. When the cells were confluent, a wound was made in the monolayer. The medium and debris were removed, and fresh medium was added to each well. Photographs of the wounded area were taken at specific time intervals, and the extent of cell migration was assessed by observing the rate at which the wound closed.

Cell migration was also assessed in Corning Transwell Permeable Supports with polycarbonate membranes $(6.5 \mathrm{~mm}$, $8-\mu \mathrm{m}$ pore size). The bottom surfaces of the well membranes were coated with fibronectin $(20 \mu \mathrm{g} / \mathrm{ml})$ in serum-free medium overnight. After MDA-MB-468 cells were cultured in serumfree medium overnight, they were harvested and resuspended $\left(5 \times 10^{5}\right.$ in $\left.250 \mu 1\right)$ in $1 \%$ BSA serum-free media and plated into the upper chambers of the transwell plates. The lower chamber of each well was filled with complete medium (10\% FBS) containing $5 \mu \mathrm{g} / \mathrm{ml}$ of fibronectin as an adhesive substrate. Cells were incubated $8 \mathrm{~h}$, fixed and stained using a DIF-Quick kit. Non-migrating cells were removed with cotton swabs. Migrating cells were enumerated in four randomly chosen fields using a x 25 objective lens of a light microscope. 
Table I. Plant lectins used in this study and their binding specificity.

\begin{tabular}{|c|c|}
\hline Lectin & Specificity \\
\hline Arachis hypogaea (PNA) & Galß(1,3)GalNAc [Thomsen Friedenreich (TF)-antigen] \\
\hline Lotus tetragonolobus (Lotus) & Terminal $\alpha$ fucose; $\operatorname{Le}^{(\mathrm{x})} \operatorname{Gal} \beta(1,4) \mathrm{Fuc} \alpha(1,3) \mathrm{GlcNAc}$ \\
\hline Sambucus nigra (SNA) & NeuAc $\alpha(2,6) \mathrm{Gal} ; \operatorname{NeuAc} \alpha(2,6) \mathrm{GalNAc}$ \\
\hline Maackiaamurensis (MAA) & $\operatorname{NeuAc} \alpha(2,3)$ Galß$(1,4)$ GlcNAc \\
\hline Erythrina cristagalli (ECL) & Galß $(1,4)$ GlcNAc \\
\hline Ulex europaeus (ULEX) & Fuc $\alpha(1,2) ;$ Fuc $\alpha(1,2) \operatorname{Gal} \beta(1,4)$ GlcNAc \\
\hline Phaseolus vulgaris (PHA-L) & Trianternary and tetraanternary complex oligosaccharides; $\beta(1,6)$ GlcNAc \\
\hline
\end{tabular}

Cell invasion assay. The Matrigel 'chemoinvasion' assay was used to study tumor cell invasion of basement membranes (21). In brief, 80-90\% confluent tumor cells were detached, washed, and resuspended in serum-free media. Then, $2 \times 10^{5}$ cells were seeded into the upper chamber of 12-mm-diameter transwells coated with Matrigel basement membrane matrix diluted to $1 \mathrm{mg} / \mathrm{ml}$ with ice-cold serum-free medium. After incubation at $37^{\circ} \mathrm{C}$ for $24 \mathrm{~h}$, the non-migrating cells on the upper surface of the filters were removed with a cotton swab. The cells migrating to the lower side of the wells were stained with DIF-Quick stain and counted by microscopy using 8 fields per treatment replicate. A Matrigel invasion index was calculated as follows: invasion index $=\%$ invasion test cells $/ \%$ invasion control cells.

F-actin staining. Staining of filamentous or F-actin with rhodamine phalloidin was performed to observe actin filament organization, as previously described (22). In brief, $1 \times 10^{3}$ cells were seeded onto coverslips placed in 6-well plates. After 2 days in culture, the media were removed, and the coverslips containing the cells were washed with PBS; permeabilized with a mixture 10X PBS, Triton X-100, and paraformaldehyde; and stained. Cells were visualized and photographed with an Olympus BX60 fluorescent microscope. Cells in randomly selected fields were counted and scored according to the presence or absence of staining of the cell periphery.

Oligonucleotide microarray studies. The three cell culture media were tested in three independent experiments. Confluent monolayers of MDA-MB-468 cells were washed once with sterile phosphate-buffered saline (PBS). RNA was extracted using TRIzol reagent per manufacturer's instructions.

The RNA sample quality was checked with an Agilent Bioanalyzer (Agilent Technologies, Palo Alto, CA). The Affymetrix GlycoV4 custom oligonucleotide array custom GeneChip (Affymetrix, Santa Clara, CA) was designed for the Consortium for Functional Glycomics (http://www. functionalglycomics.org/static/consortium/consortium.shtml). The focused array includes probes for 1260 human probe-ids related to glyco-genes. It does not contain mismatched probes. RNA from each cell lysate was labeled using the MessageAmp II-Biotin Enhanced Amplification kit (Ambion Inc, Austin, TX). Hybridization and scanning to the GlycoVv4 chip were performed according to Affymetrix-recommended protocols.
The chips were scanned using the Affymetrix GeneChip Scanner 3000. Raw expression values were normalized using RMA or Robust Multichip Average expression summary (http://rmaexpress.bmbolstad.com/). All further processing of the data was performed within the Bioconductor project and the R program software (Free Software Foundation's GNU General Public License).

Statistical analysis. Differences in fluorescent staining of F-actin were tested by the Fisher's exact test. The HUVEC adhesion and Matrigel invasion assays were analyzed with randomized block ANOVA. For each assay, the ANOVA was followed by pairwise comparison of treatment means, with p-values computed with Tukey's multiple-comparison procedure. Data are presented as means \pm SD. All statistical tests were 2-sided. Differences were considered to be statistically significant at $\mathrm{p}<0.05$. SAS software version 9.1 (SAS Institute, Cary, NC) was used for all statistical analyses.

\section{Results}

The cell-surface glycomic phenotype was modified by the culture medium sugar composition. We examined alterations in cell surface glycosylation pattern after exposure of tumor cells to fructose or glucose as the carbon source by using several plant lectins. Plant lectins have been used extensively for characterization of cell surface carbohydrate antigens and specificity of lectins that were used in this study is shown in Table I. Compared to the MDA-MB-468 cells cultured in glucose, cells grown in a 1:1 glucose:fructose ratio (glucose/fructose), or $4: 1$ fructose:glucose ratio (fructose) displayed multiple changes in lectin binding, suggesting alterations in their glycomic phenotype, i.e., their glycan structures or composition (Table II). The ThomsenFriedenreich (TF) antigen-specific lectins PNA was more reactive with cells grown in fructose, indicating an increase in the TF antigen when the glucose is partially replaced with a similar hexose. A decrease in fucosylated structures, such as $\operatorname{Le}^{\mathrm{X}}[\mathrm{Gal}(1,4) \mathrm{Fuc} \alpha(1,3) \mathrm{GlcNAc}]$ was confirmed by the decreased reactivity of the Lotus lectin with cells in fructose. ULEX binding was higher in fructose/glucose mix and lower in fructose culture, the ULEX lectin binds to terminal Fuca $(1,2)$ as in the Fuc $\alpha(1,2)$ Galß $(1,4)$ GlcNAc oligosaccharide. SNA and MAA displayed higher reactivity in glucose/fructose 
Table II. Mean fluorescence intensities of flow cytometric analyses of binding of indicated lectins to human MDA-MB-468 grown in the media supplemented with indicated carbon source.

\begin{tabular}{llllrrrr}
\hline Carbon source & PNA & Lotus & SNA & MAA & ECL & ULEX & PHA-L \\
\hline Glucose & 5.68 & 26.83 & 35.45 & 33.49 & 8.27 & 10.92 & 25.08 \\
Glucose/fructose & 1.53 & 31.55 & 66.9 & 48.09 & 4.87 & 23.51 & 27.51 \\
Fructose & 38.48 & 10.69 & 20.82 & 6.98 & 46.86 & 1.94 & 116.11 \\
\hline
\end{tabular}

culture and a drop in fructose culture, similar to ULEX reactivity. This indicates a reduction in structures containing sialic acid, NeuAcaGal; NeuAcaGalNAc, and in terminal fucosylation in fructose culture. Taken together, the data indicate that in the presence of higher proportion of fructose as a carbon source, the terminal fucose and sialic acid residues were decreased, while an increase in TF antigen was observed. It appears that sialylation may transform $\mathrm{TF}$ to sialyl TF. The higher reactivity of ECL in fructose medium points to an increase in $\operatorname{Gal}(1,4)$ GlcNAc structures.

PHA-L, a lectin that is highly reactive with trianternary and tetraanternary complex oligosaccharides, reacted more with tumor cells grown in media with added fructose relative to cells grown in glucose alone. This could signal an increase in the $\mathrm{B}-1,6 \mathrm{GlcNAc}-$ branching glycans, with poly-N-acetyl lactosamine structures, which could have functional significance in relation to adhesion proteins.

Together, these results suggest that adding fructose as a carbon source induces multiple changes in carbohydrate epitopes. Changes in glycan structures can affect proliferative, migratory, invasive and adhesive properties of tumor cells. Therefore, we examined these properties of MDA-MB-468 cells after seeding in fructose supplemented growth medium.

Carbon source affects endothelial cell adhesion. Tumor cell attachment to vascular endothelial cells is a crucial step in metastasis. The effect of introducing fructose as a carbon source on selectin-mediated adhesion to HUVECs was determined because of the increased expression of sialylated structures in fructose-treated cells. E- and P-selectin are expressed on the endothelial cells and their interaction with sialylated oligosaccharides such as $\mathrm{sLe}^{\mathrm{a}}$ and $\mathrm{sLe}^{\mathrm{x}}$ on tumor cells can lead to a higher metastatic rate (23-25). Tumor cell adhesion to activated endothelial cells is shown in Fig. 1. Overall, the treatments produced significantly different binding of the MDA-MB-468 ( $\mathrm{p}=0.025)$ to HUVECs. There was significantly increased binding $(\mathrm{p}=0.022)$ when cells were cultured at the higher fructose concentration compared with the medium containing glucose alone (Fig. 1).

Fructose accelerates tumor cell migration. Cellular motility is an ex vivo measure of cell metastatic potential (21). Migration is a multifaceted phenomenon that requires successive adhesion to and detachment from the extracellular matrix. The wound-healing assay was used to evaluate cell motility, which involves integration of biochemical and physical mechanisms. Photographs representative of three woundhealing assays conducted are shown in Fig. 2A. The wound-

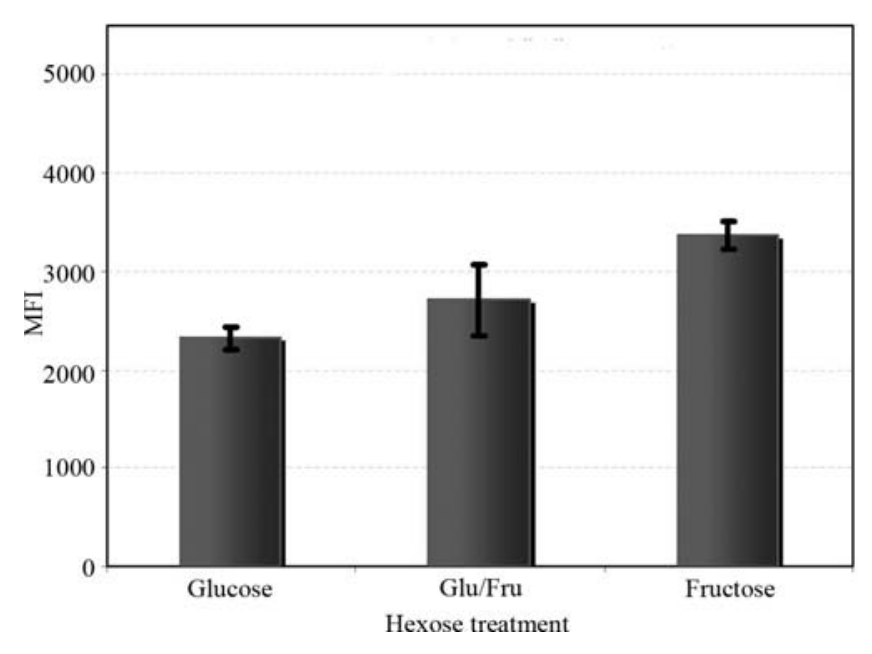

Figure 1. The sugar composition of the cell culture medium mediated the binding of Calcein fluorescent probe-labeled MDA-MB-468 cells to HUVECs, as measured by mean fluorescence intensity (MFI). Based on three independent replicated assays, cells cultured in glucose, glucose: fructose, and fructose showed MFI values of $2321( \pm 120), 2707( \pm 358)$ and 3367 $( \pm 133)$, respectively. Block ANOVA (blocking on assay replication) analysis showed a statistically significant difference in the MFI values among the carbon sources $\left(\mathrm{F}_{2,4}=10.73, \mathrm{p}=0.025\right)$, with the largest difference found between cells cultured in glucose versus fructose (Tukey-adjusted $\mathrm{p}=0.022$ ).

healing assays revealed that the sugar content of the cell culture medium had an effect on cell migration. The observed acceleration in wound closure when fructose was added to the medium could have happened because of an increase in cell proliferation or actual migratory capability. We examined cell proliferation and migration (Fig. 2B and C). The addition of fructose as the source of carbon atoms inhibited cellular proliferation but promoted migratory ability. Therefore, the faster wound closure seen in fructose-fed cells was mostly due to an increase in cell migration and not cell proliferation.

Effects of fructose on cellular morphology and F-actin staining pattern. There were distinctive changes in the shape of the MDA-MB-468 cells after 21 days of adaptation to the fructose medium (Fig. 3A). These cells took on a fibroblast-like shape and began to lose contact with neighboring cells. This is consistent with the 'epithelial to mesenchymal transition', whereby tumor cells lose their epithelial cell features and assume the more migratory and invasive attributes of mesenchymal cells. MDA-MB-468 cells cultured in either glucose or glucose/fructose maintained their rounded, cluster conformation. 


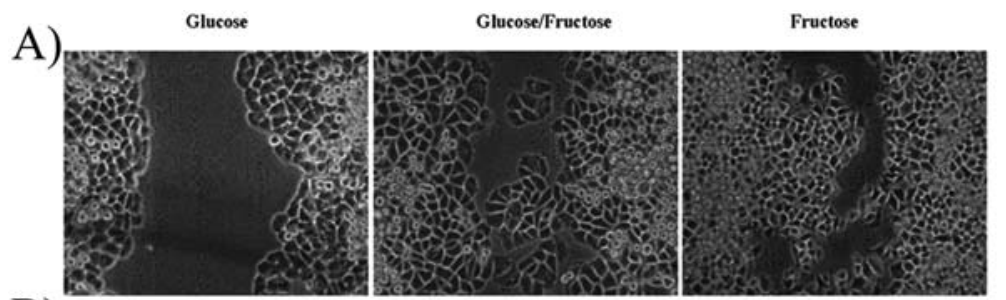

B)

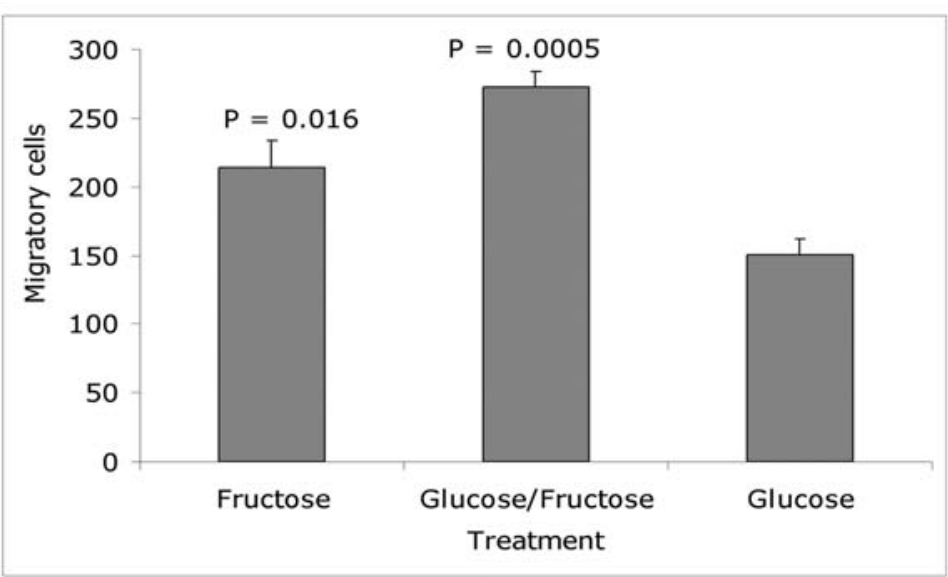

C)

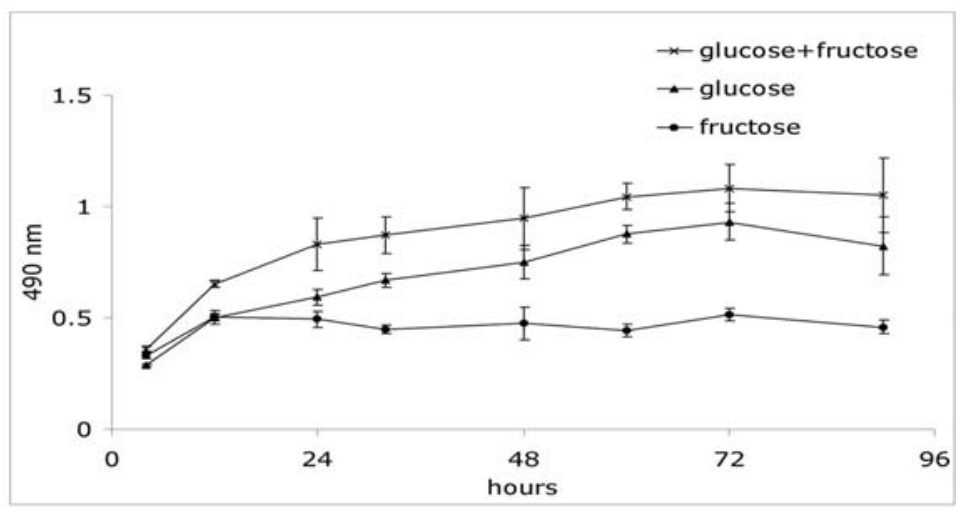

Figure 2. (A) Comparison of migratory potential of MDA-MB-468 cells in a wound-healing assay illustrated that substitution of fructose for glucose for part or most of the medium's carbon source accelerated cell migration. Phase-contrast microscopic images were taken at day 5 after wounding the surface; photographs are representative of two experiments. (B) The cell migration performed in 24-well transwell plates with 8- $\mu$ m pore size polycarbonate filters as described in Materials and methods. Briefly, following incubation for $8 \mathrm{~h}$ the cells were fixed and stained with Diff-Quick staining solution. Migrated cells were enumerated under a light microscope in a blinded fashion, based on six randomly chosen fields. Error bars were calculated based on three replications. Paired Student's t-test was used to compare cells fed with fructose or fructose/glucose with glucose only. The assay was repeated twice with similar results. (C) In vitro proliferation assay of cells fed with fructose, glucose, and fructose/glucose was performed using CellTiter 96 Aqueous One Solution (Promega, Madison, WI) according to the manufacturer's instructions. Briefly, 10x $10^{3}$ cells were cultured in wells of 96-well plates, and every $12 \mathrm{~h}$ the provided solution was added to each plate containing replicated wells for each treatment group. Plates were incubated for $1 \mathrm{~h}$ at $37^{\circ} \mathrm{C}$ and read by an ELISA reader at $490 \mathrm{~nm}$. Cell cultures were closely monitored and maintained. The assay was performed every $12 \mathrm{~h}$ until $90 \mathrm{~h}$ after seeding, when cells reached confluence. Error bars were calculated based on four replications. The assay was repeated twice with similar results.

Alterations in MDA-MB-468 cellular morphology and interactions with other cells led to speculation that changes in cell shape might be associated with changes in cytoskeletal organization. Because actin filaments supply the basic structure for maintaining cell morphology and contribute to functions such as adhesion and motility, we evaluated F-actin staining in the MDA-MB-468 cells. The cells propagated in fructose exhibited a pattern of staining for F-actin distinct from that of cells propagated in glucose (Fig. 3B). The staining revealed a dramatically altered pattern of F-actin, which is consistent with changes in F-actin cytoskeletal organization. The changes in F-actin was clearly visualized in the cells propagated in fructose versus glucose and scored as positive or negative for rhodamine phalloidin staining in 8 fields per treatment $(\mathrm{n}=1252$ cells, Fisher's exact test, $\mathrm{p}<0.0001$ ). This finding is consistent with the decline in cellular adhesion as cells become invasive. The varied morphology and shift in F-actin localization gave rise to questions about adhesion molecules, such as E-cadherin. We examined the expression of vimentin and E-cadherin in extracts of cells cultured in the test media but observed no significant differences (data not shown).

Invasive potential is increased by fructose. The invasion assays were performed after the modifications in the cellular 

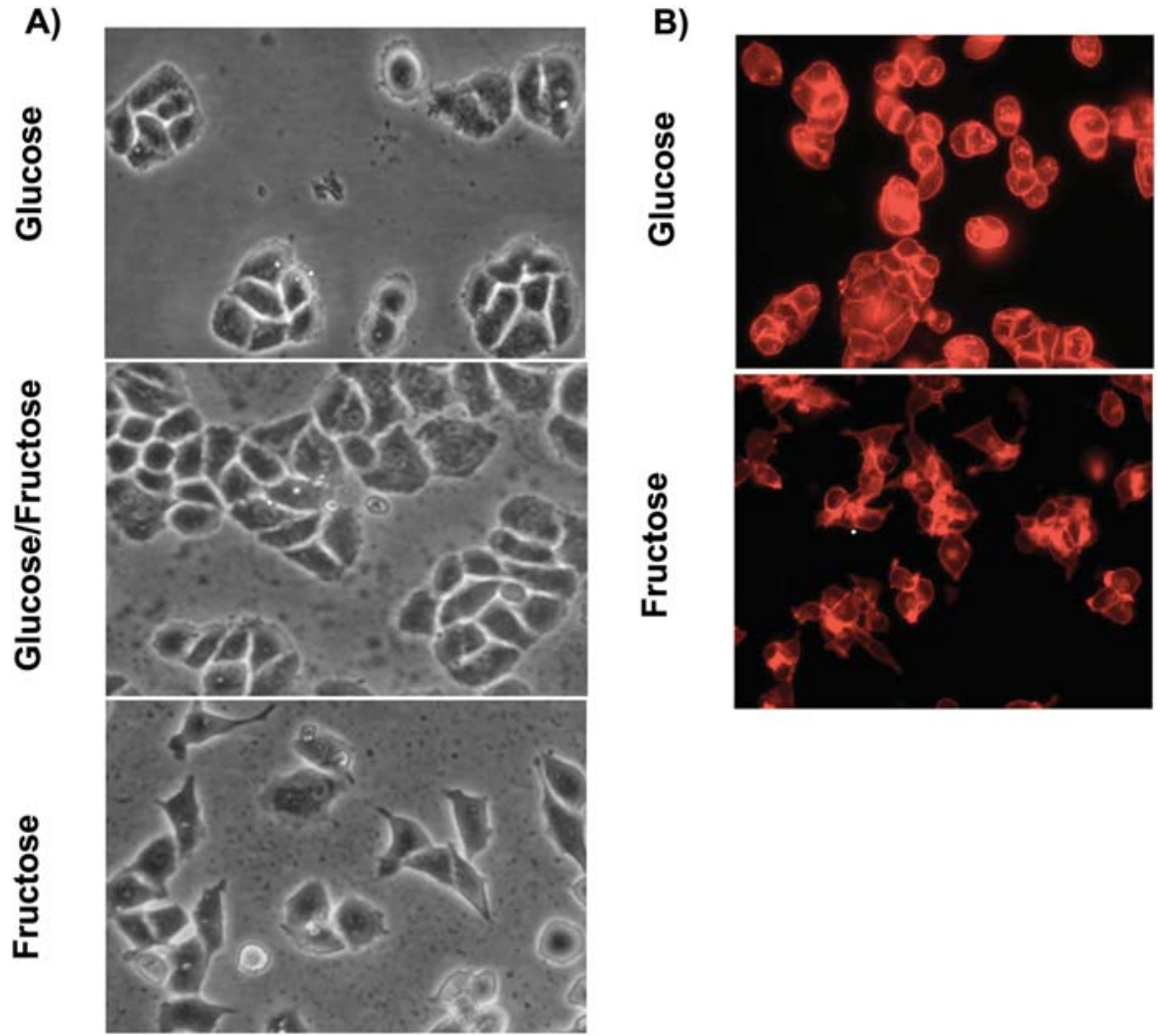

Figure 3. (A) Phase contrast microscopy was used to monitor cells for morphological changes associated with modifying the hexose composition of the culture medium. After 3 weeks, the MDA-MB-468 cells cultured in the fructose-only medium acquired an elongated morphology and displayed a more dispersed growth pattern than cells propagated in glucose or glucose: fructose. In these two media, the cells grew in cobblestone clusters. The timing of changes in cell shape was observed in three independent experiments. (B) Because morphological changes are often related to remodeling of the cytoskeleton, the actin structure of cells was visualized by rhodamine phalloidin fluorescence, using the x40 objective of a Nikon Labophot microscope. Cells cultured in the higher concentration of fructose displayed markedly less actin staining around the cell periphery. F-actin staining was further assessed by scoring for the presence or absence of intense fluorescent staining on the cell periphery in randomly selected microscopic fields on each coverslip. Of the 771 cells cultured in $25 \mathrm{mM}$ glucose, 692 (89.85\%) were intensely stained, compared to only 23 (4.8\%) of 481 cells grown in high fructose medium (Fisher's exact test, $\mathrm{p}<0.0001)$.

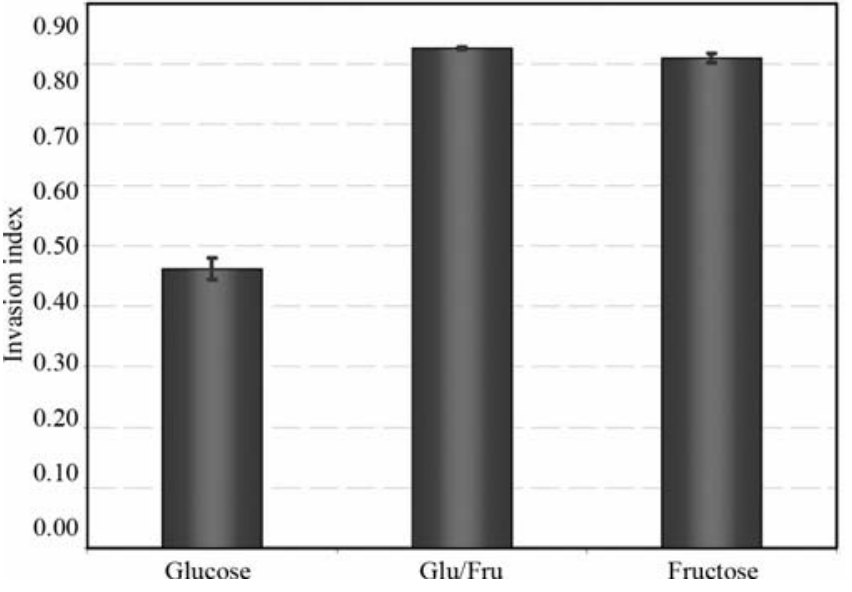

Figure 4. The Matrigel chemoinvasion assay was used to assess invasion into basement membranes. The results of two replications of the invasion assay gave similar results (ANOVA trial $\mathrm{p}=0.17$, and hexose $\mathrm{p}=0.0007$ ). Data demonstrated that using fructose as the primary carbon source significantly increased invasion of tumor cells through the basement membrane. Cells cultured in the higher fructose treatment or in fructoseglucose were more invasive than those cultured in glucose.

morphology of the MDA-MB-468 cells cultured in the higher concentration of fructose were apparent. Compared to cells grown in glucose alone, cells cultured in the presence of fructose were more invasive (Fig. 4), showing a higher invasion index in glucose/fructose $(\mathrm{p}=0.0008)$ and in the higher fructose medium $(\mathrm{p}=0.0009)$. The two replications of these invasion assays yielded similar results, with an ANOVA value of $p=0.0007$ for the hexose effect. These results indicate that the presence of fructose in the medium mediates tumor cell invasiveness.

\section{Discussion}

This study investigated the effects of introducing fructose as a carbon source in cell culture media on the phenotypic characteristics of a prototypic basal-like human breast cancer cell line that is noted to highly express the fructose transport protein GLUT 5 (16). The observed changes in phenotype are characteristics that are associated with more aggressive tumor cell lines. They included modifications in N-linked, cell-surface carbohydrate structures, combined with altered adhesive, migratory, and invasive characteristics. The results infer that exposing human breast tumor cells to a fructose-rich microenvironment might remodel the glycan architecture, enhancing tumor cell ability to metastasize more efficiently to distant sites.

The data extend the observations of others who demonstrated that using alternative carbon sources in media can lead to changes in cell size and morphology $(8,9,26)$. It has 
been reported previously that substituting other sugars for glucose in cell culture media may influence cell differentiation, sugar nucleotide pools, and gene expression (26-28). Although it seemed likely that the addition of fructose might modify the expression of genes controlling cell surface glycomic phenotype, none were observed in our microarray studies (data not shown). The lack of changes in expression of genes included in the Glyco gene probe set suggests that the fructose-triggered phenotypic changes involve post-translational events. It does not rule out up- or down-regulation of expression of other genes, especially genes expressing core proteins of proteoglycans or glycoproteins. Taken together, these results invite speculation that glycosylation or glycation of macromolecules could vary as a function of exposure to exogenous sugars. Berger et al (6), suggested that dietary sugars have novel roles in regulating glycosylation in humans. The conclusion was based on their findings that single doses of orally administered, stable isotope-labeled galactose and mannose were readily incorporated into glycans, even in the presence of glucose (6). A link between exogenous sugars and glycan structures is that carbon atoms derived from catabolism of exogenous carbohydrates are used in the biosynthesis of certain glycosyltransferase substrates, including sialic acids, $\mathrm{N}$-acetylglucosamine (GlcNAc), and $\mathrm{N}$-acetylgalactosamine (GalNAc).

Tumor cell glycans are often structurally and functionally abnormal, but the mechanisms underlying changes in glycosylation remain largely obscured. Structural variability of glycans is dictated by tissue-specific regulation of glycosyltransferase genes, availability of sugar nucleotides, and competition between enzymes for acceptor intermediates during glycan elongation (29). Structural modifications of the oligosaccharides on the surface of tumor cells can alter glycoprotein-binding characteristics (30). The presence of linear and branched poly- $\mathrm{N}$-acetyllactosamines on $\mathrm{N}$-glycans similar to those induced by fructose in this study is commonly associated with invasive and metastatic tumor cells (29). A greater abundance of $31,6-$ GlcNAc-branched glycans was recently demonstrated in metastatic lesions versus primary tumors of breast cancer patients (31). Highly elevated $B 1,6$ branching in human fibrosarcoma cells is known to modify cell matrix adhesion and migration via modulation of integrin clustering and related signal transduction pathways (32).

Variations in oligosaccharide structures contribute to folding, stability, and biologic function of glycoproteins (33). The importance of specific glycosylation events in mammary cancer metastases has been clearly demonstrated in transgenic mice (2). While the mechanisms explaining the changes detected in human breast tumor cells are presently unclear, several alternatives exist. A possible mechanism could be posttranslational modifications in protein glycosylation initiated indirectly by fructose via disruption of metabolic pathways. Because fructose is isomerized to glucose-6-phosphate, it potentially has multiple metabolic fates in the glycolytic, pentose, and glycogenesis pathways. The sustained presence of fructose may trigger changes in flux in these interrelated pathways that could alter cell surface glycomic phenotype and cell behavior. Alternatively, as a highly effective glycating agent, fructose may contribute to formation of advanced glycation end-products (34).
Together, these novel data demonstrate that adding fructose as the carbon source in growth media for a human breast tumor cell line results in changes in phenotypic characteristics that are associated with tumor progression and metastasis. However, the data must be interpreted cautiously because important questions, such as, are these findings generalizable to other breast tumor cell lines, remain to be answered.

In light of worldwide use of a novel sugar containing free fructose (35-37), our findings could have important implications for human cancers. Many of the risk factors associated with cardiovascular disease and Type 2 diabetes are also associated with breast cancer. In particular it has been proposed that sugar intake, and particularly that of dietary fructose, may have an important participatory role in the current cardiorenal disease epidemic and may also explain why certain subgroups, such as African Americans, are particularly prone to cardiovascular disease (19). Fructose is purported to be part of a biochemical process that leads to weight gain and other precursors of Type 2 diabetes. A fructose-rich diet might promote aggressive phenotypes of breast tumor cells, which might contribute to poor outcome for African Americans, as this group already has a tumor phenotype that is challenging therapeutically.

\section{Acknowledgments}

This work was supported in part by an NIH grant (CA089480) to T.K.E. and an ACS-IRG pilot grant (271/G1-11262-01E) to T.L.W., and the Breast Cancer Research Program at the Winthrop P. Rockefeller Cancer Institute. The authors are grateful for the contributions of Naresh Pemmaraju and Natalie Brush-Strode, whose work was supported by (CA049425 to T.K.). We appreciate the use of the Consortium for Functional Glycomics' Glyco-gene microarray analysis resource.

\section{References}

1. Hakomori S: Tumor-associated carbohydrate antigens defining tumor malignancy: basis for development of anti-cancer vaccines. Adv Exp Med Biol 491: 369-402, 2001.

2. Couldrey $\mathrm{C}$ and Green JE: Metastases: the glycan connection. Breast Cancer Res 2: 321-323, 2000.

3. Gorelik E, Galili U and Raz A: On the role of cell surface carbohydrates and their binding proteins (lectins) in tumor metastasis. Cancer Metastasis Rev 20: 245-277, 2001.

4. Kawaguchi T: Cancer metastasis: characterization and identification of the behavior of metastatic tumor cells and the cell adhesion molecules, including carbohydrates. Curr Drug Targets Cardiovasc Haematol Disord 5: 39-64, 2005.

5. Lee RT, Lauc G and Lee YC: Glycoproteomics: protein modifications for versatile functions. Meeting on glycoproteomics. EMBO Rep 6: 1018-1022, 2005.

6. Berger V, Perier S, Pachiaudi C, Normand S, Louisot P and Martin A: Dietary specific sugars for serum protein enzymatic glycosylation in man. Metabolism 47: 1499-1503, 1998.

7. Delhotal B, Lemonnier F, Couturier M, Wolfrom C, Gautier M and Lemonnier A: Comparative use of fructose and glucose in human liver and fibroblastic cell cultures. In Vitro 20: 699-706, 1984.

8. Wolfrom C, Loriette C, Polini G, Delhotal B, Lemonnier F and Gautier M: Comparative effects of glucose and fructose on growth and morphological aspects of cultured skin fibroblasts. Exp Cell Res 149: 535-546, 1983.

9. Wolfrom C, Kadhom N, Raulin J, Raynaud N and Gautier M: Fructose-induced enhanced mitogenicity of diploid human cells: possible relationship with cell differentiation. In Vitro Cell Dev Biol Anim 30A: 263-268, 1994.

10. Medina RA and Owen GI: Glucose transporters: expression, regulation and cancer. Biol Res 35: 9-26, 2002. 
11. Zamora-Leon SP, Golde DW, Concha, II, Rivas CI, DelgadoLopez F, Baselga J, Nualart F and Vera JC: Expression of the fructose transporter GLUT5 in human breast cancer. Proc Natl Acad Sci USA 93: 1847-1852, 1996.

12. Grover-McKay M, Walsh SA, Seftor EA, Thomas PA and Hendrix MJ: Role for glucose transporter 1 protein in human breast cancer. Pathol Oncol Res 4: 115-120, 1998.

13. Enzmann H, Ohlhauser D, Dettler T and Bannasch P: Enhancement of hepatocarcinogenesis in rats by dietary fructose. Carcinogenesis 10: 1247-1252, 1989.

14. Yam D, Fink A, Nir I and Budowski P: Insulin-tumour interrelationships in thymoma bearing mice. Effects of dietary glucose and fructose. Br J Cancer 64: 1043-1046, 1991.

15. Stamp D, Zhang XM, Medline A, Bruce WR and Archer MC: Sucrose enhancement of the early steps of colon carcinogenesis in mice. Carcinogenesis 14: 777-779, 1993.

16. Godoy A, Ulloa V, Rodriguez F, Reinicke K, Yanez AJ, Garcia Mde L, Medina RA, Carrasco M, Barberis S, Castro T, Martinez F, Koch X, Vera JC, Poblete MT, Figueroa CD, Peruzzo B, Perez F and Nualart F: Differential subcellular distribution of glucose transporters GLUT1-6 and GLUT9 in human cancer: ultrastructural localization of GLUT1 and GLUT5 in breast tumor tissues. J Cell Physiol 207: 614-627, 2006.

17. Cailleau R, Olive M and Cruciger QV: Long-term human breast carcinoma cell lines of metastatic origin: preliminary characterization. In Vitro 14: 911-915, 1978.

18. Brinkley BR, Beall PT, Wible LJ, Mace ML, Turner DS and Cailleau RM: Variations in cell form and cytoskeleton in human breast carcinoma cells in vitro. Cancer Res 40: 3118-3129, 1980

19. Johnson RJ, Segal MS, Sautin Y, Nakagawa T, Feig DI, Kang DH, Gersch MS, Benner S and Sanchez-Lozada LG: Potential role of sugar (fructose) in the epidemic of hypertension, obesity and the metabolic syndrome, diabetes, kidney disease, and cardiovascular disease. Am J Clin Nutr 86: 899-906, 2007.

20. Carey LA, Perou CM, Livasy CA, Dressler LG, Cowan D, Conway K, Karaca G, Troester MA, Tse CK, Edmiston S, Deming SL, Geradts J, Cheang MC, Nielsen TO, Moorman PG, Earp HS and Millikan RC: Race, breast cancer subtypes, and survival in the Carolina Breast Cancer Study. JAMA 295: 2492-2502, 2006.

21. Albini A, Benelli R, Noonan DM and Brigati C: The 'chemoinvasion assay': a tool to study tumor and endothelial cell invasion of basement membranes. Int J Dev Biol 48: 563-571, 2004.

22. Kelly T, Yan Y, Osborne RL, Athota AB, Rozypal TL, Colclasure JC and Chu WS: Proteolysis of extracellular matrix by invadopodia facilitates human breast cancer cell invasion and is mediated by matrix metalloproteinases. Clin Exp Metastasis 16: 501-512, 1998

23. Sawada R, Tsuboi S and Fukuda M: Differential E-selectindependent adhesion efficiency in sublines of a human colon cancer exhibiting distinct metastatic potentials. J Biol Chem 269: 1425-1431, 1994.
24. Renkonen J, Paavonen T and Renkonen R: Endothelial and epithelial expression of sialyl Lewis(x) and sialyl Lewis(a) in lesions of breast carcinoma. Int J Cancer 74: 296-300, 1997.

25. Biancone L, Araki M, Araki K, Vassalli P and Stamenkovic I: Redirection of tumor metastasis by expression of E-selectin in vivo. J Exp Med 183: 581-587, 1996.

26. Paolini DHL, Gonzalez F, Colofiore J, Rossow P and Amos H: Sugar composition of mammalian cell surface membrane: a function of carbon source. In: Cell Surface Carbohydrate Chemistry. Harmon RE (ed). Academic Press, New York, 1978.

27. Mack DR, Cheng PW, Perini F, Wei S and Hollingsworth MA: Altered expression of sialylated carbohydrate antigens in HT29 colonic carcinoma cells. Glycoconj J 15: 1155-1163, 1998.

28. Malide D, Davies-Hill TM, Levine M and Simpson IA: Distinct localization of GLUT-1, -3 , and -5 in human monocyte-derived macrophages: effects of cell activation. Am J Physiol 274: E516-E526, 1998.

29. Dennis JW, Granovsky M and Warren CE: Protein glycosylation in development and disease. Bioessays 21: 412-421, 1999.

30. Guillot J, Guerry M, Konska G, Caldefie-Chezet F, De Latour M and Penault-Llorca F: [Modification of glycoconjugates during the carcinogenesis: the case of mammary carcinomas]. Bull Cancer 91: 141-158, 2004.

31. Handerson T, Camp R, Harigopal M, Rimm D and Pawelek J: Beta1,6-branched oligosaccharides are increased in lymph node metastases and predict poor outcome in breast carcinoma. Clin Cancer Res 11: 2969-2973, 2005.

32. Guo HB, Lee I, Kamar M, Akiyama SK and Pierce M: Aberrant $\mathrm{N}$-glycosylation of beta1 integrin causes reduced alpha5beta1 integrin clustering and stimulates cell migration. Cancer Res 62: 6837-6845, 2002

33. Monzavi-Karbassi B, Whitehead TL, Jousheghany F, Artaud C, Hennings L, Shaaf S, Slaughter A, Korourian S, Kelly T, Blaszczyk-Thurin $\mathrm{M}$ and Kieber-Emmons T: Deficiency in surface expression of E-selectin ligand promotes lung colonization in a mouse model of breast cancer. Int J Cancer 117: 398-408, 2005.

34. Levi B and Werman MJ: Fructose and related phosphate derivatives impose DNA damage and apoptosis in L5178Y mouse lymphoma cells. J Nutr Biochem 14: 49-60, 2003.

35. Putnam J, Allshouse J and Kantor L: U.S. per capita food supply trends: more calories, refined carbohydrates and fats. Food Review 25: 2-15, 2002.

36. Vuilleumier S: Worldwide production of high-fructose syrup and crystalline fructose. Am J Clin Nutr 58: S733-S736, 1993.

37. Romieu I, Lazcano-Ponce E, Sanchez-Zamorano LM, Willett W and Hernandez-Avila M: Carbohydrates and the risk of breast cancer among Mexican women. Cancer Epidemiol Biomarkers Prev 13: 1283-1289, 2004. 